\title{
Stochastic Models of Gene Expression with Delayed Degradation
}

\author{
Jacek Miękisz • Jan Poleszczuk • Marek Bodnar • \\ Urszula Foryś
}

Received: 25 May 2010 / Accepted: 10 December 2010 / Published online: 6 January 2011

(C) The Author(s) 2011. This article is published with open access at Springerlink.com

\begin{abstract}
In many biochemical reactions occurring in living cells, number of various molecules might be low which results in significant stochastic fluctuations. In addition, most reactions are not instantaneous, there exist natural time delays in the evolution of cell states. It is a challenge to develop a systematic and rigorous treatment of stochastic dynamics with time delays and to investigate combined effects of stochasticity and delays in concrete models.

We propose a new methodology to deal with time delays in biological systems and apply it to simple models of gene expression with delayed degradation. We show that time delay of protein degradation does not cause oscillations as it was recently argued. It follows from our rigorous analysis that one should look for different mechanisms responsible for oscillations observed in biological experiments.

We develop a systematic analytical treatment of stochastic models of time delays. Specifically we take into account that some reactions, for example degradation, are consuming, that is: once molecules start to degrade they cannot be part in other degradation processes.
\end{abstract}

Electronic supplementary material The online version of this article (doi:10.1007/s11538-010-9622-4) contains supplementary material, which is available to authorized users.

J. Miękisz $(\bowtie) \cdot$ J. Poleszczuk · M. Bodnar · U. Foryś

Institute of Applied Mathematics and Mechanics, University of Warsaw, Banacha 2, 02-097 Warsaw, Poland

e-mail: j.miekisz@mimuw.edu.pl

J. Poleszczuk

e-mail: j.poleszczuk@mimuw.edu.pl

M. Bodnar

e-mail: m.bodnar@mimuw.edu.pl

U. Foryś

e-mail: u.forys@mimuw.edu.pl 
We introduce an auxiliary stochastic process and calculate analytically the variance and the autocorrelation function of the number of protein molecules in stationary states in basic models of delayed protein degradation.

Keywords Time delay · Noise · Protein degradation - Stochastic delay equations · Systems biology

\section{Introduction}

Gene expression is a complex process involving many biochemical reactions with proteins being final products. In a deterministic approximation, it might be modeled by a system of a few differential kinetic rate equations describing transcription, translation, and degradation. It is usually assumed that all these processes are instantaneous. However, they are rather slow, for example the average transcription speed is about 20 nucleotides/s and the average translation speed is about 2 codons/s (Alberts et al. 2002). In addition, biochemical processes usually involve several sequential steps. It is a common procedure to replace these steps by one fixed time delay (Bratsun et al. 2005; Timmer et al. 2004; Roussel and Zhu 2006; Krishna et al. 2006; Tiana et al. 2007; Mather et al. 2009). Time delays might also be used to account for transport of molecules in spatial models (Marquez-Lago et al. 2010). Time delays in biological system are usually ascribed to transcription and translation (Lewis 2003; Jensen et al. 2003; Monk 2003; Bernard et al. 2006; Barrio et al. 2006). They may cause oscillations of mRNA and protein concentrations. However, the process of degradation of both mRNA (Clayton and Shapira 2007) and proteins (Clayton and Shapira 2007; Liu et al. 2000; Smolen et al. 2001; Sriram and Gopinathan 2004) can also consist of several steps and therefore can be modeled by explicit time delays. Delayed degradation of JAK2 protein in signaling pathways was discussed in Melzner et al. (2006).

Delayed protein degradation was recently studied in (Bratsun et al. 2005). It was assumed there that protein degradation is realized by a sequence of phosphorylation steps at the rate $\sim 1 \mathrm{~h}^{-1}$, while non-delayed degradation has the rate $\sim 0.3 \mathrm{~h}^{-1}$. This leads to differential equations with time delays (Györi and Ladas 1991; Gopalsamy 1992; Kuang 1993; Hale 1997; Erneux 2009). It is well known that solutions of time-delay differential equations may exhibit an oscillatory behavior: for small delays the system may approach a stable stationary point in an oscillatory manner and for large delays it undergoes the Hopf bifurcation and there appears a limit cycle. It was argued in Bratsun et al. (2005) that time delays of degradation can cause oscillations in genetic regulatory systems in a way similar to delayed transcription and translation as discussed in Lewis (2003), Monk (2003), Bernard et al. (2006), Barrio et al. (2006). The main goal of our paper is to show that the delayed protein degradation alone cannot be responsible for oscillations. Therefore we should look for different mechanisms responsible for oscillations observed in biological experiments.

In many cases, biochemical processes take place in small volumes and may involve only few molecules. Deterministic approach dealing with macroscopic concentrations of molecules is then inappropriate. A small number of molecules taking 
part in gene expression results in significant random fluctuations. To take into account such fluctuations, many stochastic models involving Master, Fokker-Planck, and Langevin equations were proposed (Berg 1978; McAdams and Arkin 1997; Kepler and Elston 2001; Thattai and van Oudenaarden 2001; Swain et al. 2002; Paulsson 2004, 2005; Hornos et al. 2005; Komorowski et al. 2009), and appropriate birth and death processes were simulated by the use of the Gillespie algorithm (Gillespie 1977).

Effects of the interplay between time delay and stochasticity on the behavior of small biochemical systems were analyzed in Bratsun et al. (2005), Barrio et al. (2006), Tian et al. (2007), Galla (2009), for a recent review of delayed stochastic models of gene expression and regulation see Ribeiro (2010). Theoretical foundations of stochastic delay equations were discussed in Mao et al. (2005), Lei and Mackey (2007) and validations of algorithms for delayed stochastic simulations in Roussel and Zhu (2006) and Schlicht and Winkler (2008) (the authors have not taken into account that some reactions might be consuming, see below). However, constructive analytical results are still scarce. Some results were obtained for a delayed random walker in Ohira and Milton (1995), Ohira and Yamane (2000), see also Küchler and Mensch (1992), the limit of a small delay was investigated in Guillouzic et al. (1999), where an original delay system was approximated by non-delayed stochastic differential equations. In their recent paper, Bratsun et al. (2005) studied combined effects of time delays and stochasticity on the behavior of genetic regulatory networks. They assumed that the time delay is sufficiently big, truncated Master equations and derived analytical expressions for the variance and the autocorrelation function of the number of protein molecules. In many cases they observed an oscillatory behavior.

In the present work, we develop a new methodology to deal with time delays in biological systems. It is based on the division of reactions into consuming and nonconsuming ones (Barrio et al. 2006; Cai 2007). We apply it to simple gene expression models with a delayed degradation which we assume to be a consuming reaction. That is, contrary to Bratsun et al. (2005), we do not allow any protein molecule which has already started a process of a delayed degradation to take part in another degradation process. As a consequence, models with a delayed degradation do not exhibit an oscillatory behavior. We obtain analytical expressions for the variance and the autocorrelation function for any time delay. For all stochastic simulations presented in this paper we use the exact algorithm developed in Cai (2007).

\section{Methodology}

In the simplest production-degradation system, specific bio-molecules are produced and degrade with constant intensities. Let us denote by $x(t)$ the concentration of molecules at time $t$. The classical equation of chemical kinetics, i.e. the time evolution of $x(t)$ then reads

$$
\frac{d x}{d t}=k-\gamma x(t)
$$

where $k$ is the intensity of production and $\gamma$ the intensity of degradation. 
Now we take into account that the degradation process takes some time, that is, molecules cease to exist $\tau$ units of time after the delayed degradation is triggered. One is tempted to model such a phenomenon by the following time-delay differential equation (see Bratsun et al. 2005):

$$
\frac{d x}{d t}=k-\gamma x(t-\tau)
$$

However, this means that degrading molecules affect the concentration at the future time (they are not accounted yet) and in the meantime they may again take part in another process of degradation. Therefore they may be subtracted from the system several times and this may make the concentration negative at some time. This is a frequent problem that solutions of time-delay differential equations with positive initial conditions may become negative (Bodnar 2000). From a biological point of view such models are therefore not acceptable.

We propose here a new methodology to deal with time delays in biological systems. When a molecule starts to degrade then we consider it inactive (it cannot take part in another reaction) but it is still in the system and hence it is visible. Such reactions are called consuming (Barrio et al. 2006; Cai 2007). This is a general situation but in certain cases degrading molecules may still perform some functions, for example receptors may still convey signals. Problems connected with consuming reactions were already discussed in Barrio et al. (2006). However, no algorithm was implemented there because all considered reactions were non-consuming. In Cai (2007), a general Gillespie-type algorithm appropriate for consuming reactions was designed and applied to a simple second-order reaction.

We denote by $x$ the concentration of active molecules and by $y$ the concentration of all molecules present in the system. We arrive at the following equations for $x$ and $y$ :

$$
\begin{aligned}
& \frac{d x}{d t}=k-\gamma x(t), \\
& \frac{d y}{d t}=k-\gamma x(t-\tau) .
\end{aligned}
$$

A similar approach was introduced in Marquez-Lago et al. (2010) to model spatial stochastic processes by deterministic systems with time delays. Buffer variables were introduced to keep track of molecules not available for other reactions. However, in order to fit the data obtained from stochastic simulations, different rates were used to increase and decrease the state of the buffer variables.

Now we may solve (3a), substitute the solution into (3b) and solve it with the known delayed function $x(t-\tau)$, see later (9) and (27). Equation (3b) is not really a timedelay differential equation - the delay in (3b) is in $x$ not in $y$. Therefore, the Hopf bifurcation is absent and we may not expect an oscillatory behavior while approaching an asymptotically stable stationary point. We see that the effect of time delay on the behavior of biological dynamical systems may depend on specific mechanisms of delays and we should take this into account in modeling time delays. The effect of time delays on the long-run behavior of population replicator dynamics of social and 
biological type was recently investigated in Alboszta and Miękisz (2004), Miękisz (2008). Certain biological constraints caused there the stability of stationary points and the absence of oscillations as opposed to the social model with oscillations. Below we will discuss in detail time-delay equations which appear in simple models of gene expression.

Microscopic analogs of (3), describing systems with a small number of molecules and taking into account stochastic fluctuations inherent in such systems are based on birth and death processes with appropriate probabilities corresponding to deterministic intensities $k$ and $\gamma$. Our main assumption, which is that degradation is a consuming reaction, implies that a molecule which starts to degrade is no longer active and as a consequence the stochastic process describing the evolution of the number of active molecules is the standard birth and death process (no delays are present) with the Poisson stationary distribution. The process describing the evolution of the total number of molecules is more complex. Exploiting properties of the underlying evolution of the number of active molecules, we are able to obtain various characteristics of the second process such as the variance and the autocorrelation function.

\section{Models and Results}

\subsection{Delayed Protein Degradation—a Reduced Model}

\subsubsection{Deterministic Description}

In a classical model of gene expression (Thattai and van Oudenaarden 2001), molecules of mRNA are produced from DNA in the process of transcription and then give rise to production of protein molecules in the process of translation. Both types of molecules may degrade. We denote intensities of above biochemical reactions by $k_{r}, k_{p}, \gamma_{r}$, and $\gamma_{p}$, respectively,

$$
\begin{aligned}
& \text { DNA } \stackrel{k_{r}}{\longrightarrow} \text { mRNA, } \\
& \text { mRNA } \stackrel{k_{p}}{\longrightarrow} P, \quad P \stackrel{\gamma_{p}}{\longrightarrow} \emptyset .
\end{aligned}
$$

We denote concentrations of mRNA and proteins by $x$ and $z$, respectively, and then the standard equations of chemical kinetics read

$$
\begin{aligned}
& \frac{d x}{d t}=k_{r}-\gamma_{r} x(t), \\
& \frac{d z}{d t}=k_{p} x(t)-\gamma_{p} z(t) .
\end{aligned}
$$

To simplify even further our model, we assume that the transcription is much faster than translation and therefore we may consider mRNA to be in a stationary state, $x_{\mathrm{st}}=k_{r} / \gamma_{r}$. We arrive at the so-called Kepler-Elston approximation (Kepler and Elston 2001), where transcription and translation are lumped into one process and 
Fig. 1 Comparison of numerical solution of (7) (solid line) and a mean trajectory obtained by Gillespie stochastic simulations of (6) (dots) without taking into account that degradation is consuming. Parameters are fixed at $k=100$, $\gamma=1.3, \gamma_{d}=1, \tau=2$

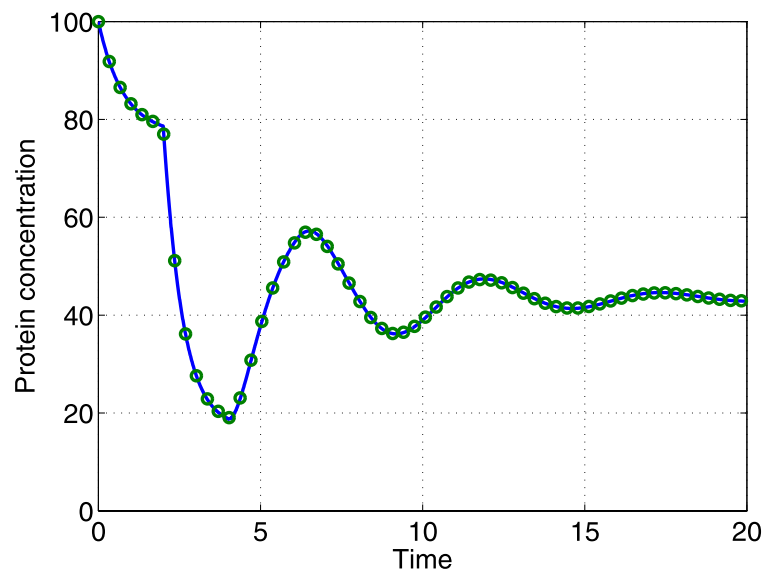

it seems that proteins are produced directly from DNA with the rescaled intensity $k_{p} x(t) \rightarrow k_{p} k_{r} / \gamma_{r} \equiv k$. We rename now $z$ as $x$, set $\gamma_{r} \equiv \gamma$ and hence the evolution of the concentration of protein molecules follows (1).

Following Bratsun et al. (2005), we take into account that the process of protein degradation consists of several steps which take certain time and we model it by a single process with time delay $\tau$ and intensity $\gamma_{d}$ (we keep the instantaneous degradation with intensity $\gamma$ ):

$$
\text { DNA } \stackrel{k}{\rightarrow} P, \quad P \stackrel{\gamma}{\rightarrow} \emptyset, \quad P \stackrel{\gamma_{d}}{\Longrightarrow} \emptyset .
$$

Bratsun et al. (2005) proposed the following time-delay differential equation to describe the time evolution of such system:

$$
\frac{d x}{d t}=k-\gamma x(t)-\gamma_{d} x(t-\tau)
$$

All the problems discussed in the Methodology section apply to this equation. To solve it, we have to specify an initial condition on the whole interval of the length $\tau$. In the case of reactions starting at $t=0$, the initial data may have the following form:

$$
x_{0}(t)=0 \quad \text { for } t<0, \quad x_{0}(0)=x^{0}>0 .
$$

Equation (7) has one stationary point $x^{\star}=\frac{k}{\gamma+\gamma_{d}}$ and it is well known that for $\gamma_{d}>\gamma$ there exists a critical value of the delay $\tau_{\mathrm{cr}}=\arccos \left(-\gamma / \gamma_{d}\right) /\left(\sqrt{\gamma_{d}^{2}-\gamma^{2}}\right)>0$ at which the Hopf bifurcation occurs, that is, the stationary point loses its stability and there appears a limit cycle, which is locally asymptotically stable (compare e.g. Györi and Ladas 1991; Hale 1997; Foryś 2004). Below $\tau_{\mathrm{cr}}$, the stationary point is asymptotically stable but it is approached in an oscillatory manner, see Fig. 1. However, as we have mentioned earlier, solutions of time-delay equations can become negative even for non-negative initial data (Bodnar 2000). In fact, it can be shown that for $\gamma_{d}>\gamma$ and $\tau>\tau_{\mathrm{cr}}$, that is, for parameters for which stable oscillations occur, solutions of (7) 
become negative. Therefore (7) cannot really describe any biochemical process and hence delay-induced oscillations present in its solutions are not biologically relevant.

Now, following our methodology, we assume that a protein that has started the process of a delayed degradation cannot participate in any other degradation process. We generalize (3) and propose the following system of equations:

$$
\begin{aligned}
& \frac{d x}{d t}=k-\left(\gamma+\gamma_{d}\right) x(t), \\
& \frac{d y}{d t}=k-\gamma x(t)-\gamma_{d} x(t-\tau),
\end{aligned}
$$

with the initial data $x_{0}(t)=y_{0}(t)=0$ for $t<0$ and $x_{0}(0)=y_{0}(0)=x^{0}$, where $x(t)$ denotes the concentration of active proteins in the system and $y(t)$ denotes the concentration of all proteins, that is, active proteins and proteins that have entered a path of a delayed degradation.

Equation (9a) is a simple linear ordinary differential equation that can be easily solved,

$$
x(t)=\frac{k}{\gamma+\gamma_{d}}+\left(x^{0}-\frac{k}{\gamma+\gamma_{d}}\right) e^{-\left(\gamma+\gamma_{d}\right) t} .
$$

It follows that for any initial value $x^{0}, x(t)$ tends to the steady state,

$$
\lim _{t \rightarrow \infty} x(t)=\frac{k}{\gamma+\gamma_{d}} .
$$

Plugging (10) into (9b) and integrating with respect to $t$, one obtains

$$
y(t)= \begin{cases}x^{0}+\gamma_{d} \bar{x} t+\frac{\gamma \bar{x}}{k}\left(x^{0}-\bar{x}\right)\left(e^{-\bar{\gamma} t}-1\right), & t \in[0, \tau], \\ x^{0}+\gamma_{d} \bar{x} \tau+\frac{\bar{x}}{k}\left(x^{0}-\bar{x}\right)\left[\gamma\left(e^{-\bar{\gamma} t}-1\right)\right. & \\ \left.\quad+\gamma_{d}\left(e^{-\bar{\gamma}(t-\tau)}-1\right)\right] & t \in(\tau, \infty),\end{cases}
$$

where $\bar{x}=\frac{k}{\gamma+\gamma_{d}}$ and $\bar{\gamma}=\gamma+\gamma_{d}$, see Fig. 2. It follows from (12) that for any initial $x^{0}$, the solution of (9b) tends to its steady state,

$$
\lim _{t \rightarrow \infty} y(t)=\frac{k}{\gamma+\gamma_{d}}\left(1+\gamma_{d} \tau\right) .
$$

This shows that for reactions (6), no oscillations are possible. In particular, time delay cannot lead to the occurrence of any, even dumped, oscillations.

\subsubsection{Stochastic Description}

In many living cells, especially prokaryotic, the number of protein molecules is rather small. To take into account random fluctuations, one has to use stochastic modeling. Biochemical reactions take place in random times and are described by birth and death processes. In such processes, probabilities of production and degradation of a single molecule are proportional to intensities of corresponding kinetic equations. 
Fig. 2 Comparison of numerical solution of (9b) (solid line) and a mean trajectory obtained by stochastic simulations of reactions given by (6) (dots) taking into account a consuming character of degradation. Parameters are fixed at $k=100, \gamma=1.3$, $\gamma_{d}=1, \tau=2$

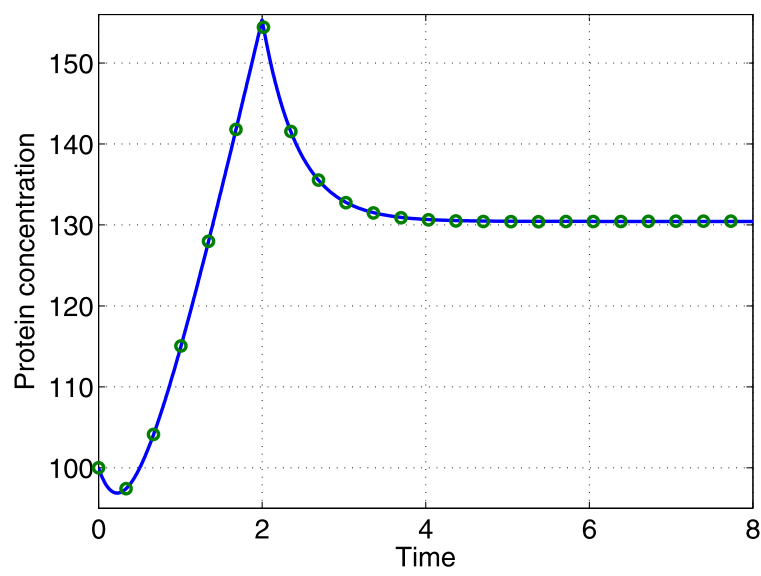

Bratsun et al. (2005) proposed the Gillespie-type algorithm (Gillespie 1977) to perform numerical simulations of birth and death processes corresponding to deterministic delay equations. To obtain analytical results they assumed that the delay $\tau$ is sufficiently big such that events at time $t-\tau$ and $t$ are decoupled. Then using the appropriately truncated Master equations and the generating function approach they were able to get an analytical expression for the autocorrelation function of the number of protein molecules. We do not assume the factorization of the joint probability distribution of the number of molecules at times $t-\tau$ and $t$. Our results are therefore valid for any time delay $\tau$.

Let $p(t)$ denote the number of active proteins, that is, proteins that are able to take part in any reaction (a delayed or an instantaneous degradation in our case) and let $d(t)$ denote the number of delayed degradations which were initiated in $[0, t)$. The total number of observed proteins $y(t)$ in the system is related to $p$ and $d$ in the following way:

$$
y(t)=p(t)+d(t)-d(t-\tau) .
$$

Now, we can write the Master equation for the probability mass function $P(p, d, t)$, that is, the probability that our system is at the state $(p, d)$ at the time $t$ (Van Kampen 1997):

$$
\begin{aligned}
\frac{d P(p, d, t)}{d t}= & k(P(p-1, d, t)-P(p, d, t)) \\
& +\gamma((p+1) P(p+1, d, t)-p P(p, d, t)) \\
& +\gamma_{d}((p+1) P(p+1, d-1, t)-p P(p, d, t)) .
\end{aligned}
$$

Our goal is to obtain the expected value $\langle y\rangle$ and the variance $\operatorname{Var}(y)$ of the total number of protein molecules $y$ and the autocorrelation function $K(T)=(\langle y(t+$ $\left.T) y(t)\rangle-\langle y(t)\rangle^{2}\right) / \operatorname{Var}(y)$ in the stationary state, that is, in the limit $t \rightarrow \infty$, when the left-hand side of (15) is equal to zero.

Let us notice that the number of active molecules follows the standard birth and death process without time delay, so in the stationary state the number of active mole- 
cules has the Poisson distribution. In the stationary state, the average number of produced protein molecules in the time interval of the length $\tau$, that is, $k \tau$, is equal to the average number of combined instantaneous degradations that were realized during that time interval and delayed degradations which were initiated during that time. The average number of delayed degradations, $\langle d(t)-d(t-\tau)\rangle$, constitutes $\gamma_{d} /\left(\gamma+\gamma_{d}\right)$ fraction of all degradations. It follows that

$$
\langle d(t)-d(t-\tau)\rangle=\frac{\gamma_{d}}{\left(\gamma+\gamma_{d}\right)} k \tau
$$

and hence we get

$$
\langle y\rangle=\langle p\rangle\left(1+\gamma_{d} \tau\right)
$$

which is fully consistent with the stationary point (13) which we obtained in the deterministic description.

For the variance of the total number of protein molecules we write

$$
\begin{aligned}
\operatorname{Var}(y(t))= & \operatorname{Var}(p(t)+d(t)-d(t-\tau)) \\
= & \operatorname{Var}(p(t))+\operatorname{Var}(d(t)-d(t-\tau)) \\
& +2 \operatorname{Cov}(p(t),(d(t)-d(t-\tau))) .
\end{aligned}
$$

Using the generating function approach to the Master equation (15) we are able to show (see the Supporting Information) that in the stationary state $\operatorname{Cov}(p(t),(d(t)-$ $d(t-\tau)))=0$ and $\operatorname{Var}(d(t)-d(t-\tau))=\langle d(t)-d(t-\tau)\rangle$ so from (16) and (18) it follows that

$$
\operatorname{Var}(y)=\langle y\rangle=\langle p\rangle\left(1+\gamma_{d} \tau\right)
$$

We see that the variance of the total number of protein molecules is equal to its mean. This may be compared to the variance obtained in Bratsun et al. (2005) which is of course independent of the time delay and is equal to $k / \gamma$. We are also able to obtain the analytical expression for the autocorrelation function (for the derivation see the Supporting Information),

$$
K(T)=\frac{1}{\operatorname{Var}(y)} \begin{cases}\frac{k\left(\gamma e^{-\bar{\gamma} T}+\gamma_{d}(1+\bar{\gamma}(\tau-T))\right)}{\bar{\gamma}^{2}}, & T \in[0, \tau], \\ \frac{k e^{-\bar{\gamma} T}\left(\gamma+\gamma_{d} e^{\bar{\gamma} \tau}\right)}{\bar{\gamma}^{2}}, & T \in(\tau,+\infty),\end{cases}
$$

where $\bar{\gamma}=\gamma+\gamma_{d}$.

In Fig. 3 we compare our autocorrelation function in our model with one obtained in Bratsun et al. (2005). In our model, the autocorrelation function monotonically decreases which indicates the lack of oscillations in the system. In Bratsun et al. (2005), we observe two peaks, a sign of oscillations. However, the authors have not taken into account the consuming character of the degradation process. Moreover, they assume 

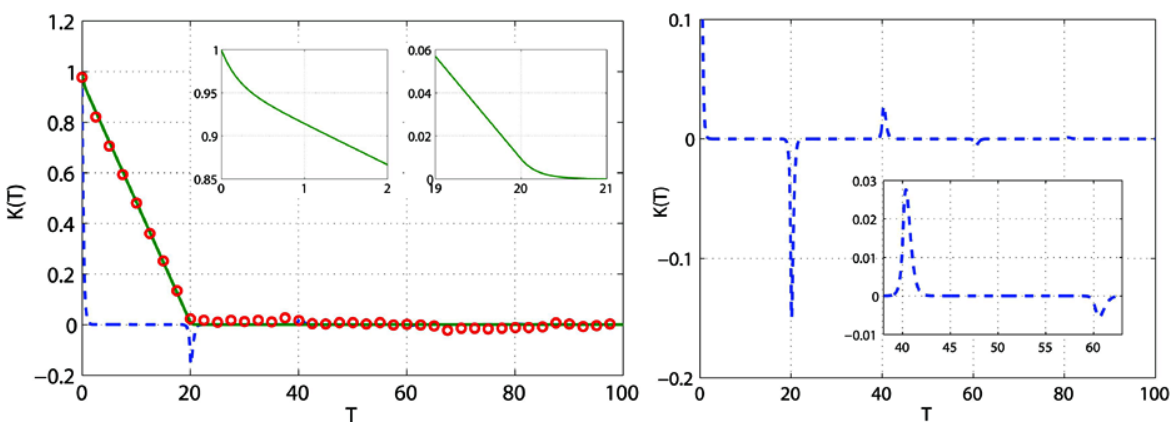

Fig. 3 Comparison of the autocorrelation function given in (20) (solid line), the one obtained by stochastic simulations (dots), and the one derived in Bratsun et al. (2005) (dashed line). Inset shows graphs of the autocorrelation given in (20) for $T \in[0,2]$ and $T \in[19,21]$. Parameters are fixed at $k=100, \gamma=4.1$, $\gamma_{d}=1, \tau=20$

that events at time $t-\tau$ and $t$ are decoupled and factor the joint probability distribution of the number of molecules at time $t-\tau$ and $t$. Then one may ask why their autocorrelation function is not equal to zero for time separations greater than or equal to $\tau$.

\subsubsection{Stochastic Delay}

Although in some cases, the time delay is very precise and fixed (like in transcription and translation), typically it is distributed around its average and may take different values with certain probabilities. Distributed random time delays were studied in Roussel and Zhu (2006), Marquez-Lago et al. (2010). One might think that distributed time delays will cause time homogenization and we will not see any effects of time delays. Below we will show that this is not the case.

We will assume now that $\tau$ is not constant but it is a random variable. To illustrate the main idea we present here the simplest case when $\tau$ may have only two values. This can be easily extended to any finite discrete distribution and then generalized to any continuous distribution with a compact support. Assume now that $\tau$ is a random variable which may have two values: $\tau_{1}$ and $\tau_{2}$ with probabilities $q_{1}$ and $q_{2}=1-q_{1}$, respectively, without the loss of generality we assume that $\tau_{1}<\tau_{2}$. We have the following reaction network:

$$
\text { DNA } \stackrel{k}{\rightarrow} P, \quad P \stackrel{\gamma}{\rightarrow} \emptyset, \quad P \stackrel{\gamma_{d} q_{1}, \tau_{1}}{\longrightarrow} \emptyset, \quad P \stackrel{\gamma_{d} q_{2}, \tau_{2}}{=} \emptyset,
$$

where $\gamma_{d} q_{i}$ and $\tau_{i}$ over the arrow denote the rate and the duration of the reaction. It can be easily seen that if the next reaction will be a delayed degradation, then the probability that the duration of the delayed degradation is equal to $\tau_{i}$ is $q_{i}$ for $i=1,2$.

The stochastic process that describes the reactions (21) is defined by the following Master equation, 


$$
\begin{aligned}
\frac{d P\left(p, d_{1}, d_{2}, t\right)}{d t}= & k\left(P\left(p-1, d_{1}, d_{2}, t\right)-P\left(p, d_{1}, d_{2}, t\right)\right) \\
& +\gamma\left((p+1) P\left(p+1, d_{1}, d_{2}, t\right)-p P\left(p, d_{1}, d_{2}, t\right)\right) \\
& +\gamma_{d} q_{1}\left((p+1) P\left(p+1, d_{1}-1, d_{2}, t\right)-p P\left(p, d_{1}, d_{2}, t\right)\right) \\
& +\gamma_{d} q_{2}\left((p+1) P\left(p+1, d_{1}, d_{2}-1, t\right)-p P\left(p, d_{1}, d_{2}, t\right)\right),
\end{aligned}
$$

where $p$ is number of active proteins, that is, proteins that are able to take part in any reaction and $d_{1}, d_{2}$ are numbers of delayed reactions with duration $\tau_{1}, \tau_{2}$, respectively, that occurred since $t=0$.

The total number of protein molecules $y(t)$ in the system is described by the following process (for $t \in\left(\tau_{2}, \infty\right)$ ):

$$
y(t)=p(t)+d_{1}(t)-d_{1}\left(t-\tau_{1}\right)+d_{2}(t)-d_{2}\left(t-\tau_{2}\right) .
$$

We may now apply our methodology to derive expressions for moments of the total number of protein molecules (see the Supporting Information). We get

$$
\operatorname{Var}(y)=\langle y\rangle=\langle p\rangle\left(1+\gamma_{d}\langle\tau\rangle\right)
$$

where $\langle\tau\rangle=q_{1} \tau_{1}+q_{2} \tau_{2}$.

We see that the expected value and the variance of the total number of protein molecules are equal as before and depend on the expected value of the delay. We are also able to obtain the analytical expression for the autocorrelation function (for the derivation see the Supporting Information),

$$
\begin{aligned}
K(T)= & \frac{1}{\operatorname{Var}(y)}\left[\tilde{K}\left(T, \gamma+q_{2} \gamma_{d}, q_{1}, \tau_{1}\right)+\tilde{K}\left(T, \gamma+g_{1} \gamma_{d}, q_{2}, \tau_{2}\right)+\bar{p} \mathrm{e}^{-\bar{\gamma} T}\right. \\
& +\frac{\bar{p} q_{1} q_{2} \gamma_{d}^{2}}{\bar{\gamma}^{2}}\left(\mathrm{e}^{-\bar{\gamma} \tau_{1}}-1\right)\left(\mathrm{e}^{-\bar{\gamma} \tau_{2}}-1\right) \mathrm{e}^{\bar{\gamma}\left(\tau_{1}+\tau_{2}-T\right)}\left(\mathrm{e}^{-2 \bar{\gamma} \max \left(\tau_{1}, \tau_{2}-T\right)}\right. \\
& \left.\left.+\mathrm{e}^{-2 \bar{\gamma} \tau_{2}}\right)\right]
\end{aligned}
$$

where

$$
\tilde{K}(T, \omega, q, \tau)= \begin{cases}\frac{\bar{p}\left(\omega e^{-\bar{\gamma} T}+q \gamma_{d}(1+\bar{\gamma}(\tau-T))\right)}{\bar{\gamma}}, & T \in[0, \tau], \\ \frac{\bar{p} e^{-\bar{\gamma} T}\left(\omega+q \gamma_{d} q e^{\bar{\gamma} \tau}\right)}{\bar{\gamma}}, & T \in\left(\tau_{i},+\infty\right),\end{cases}
$$

and $\bar{\gamma}=\gamma+\gamma_{d}, \bar{p}=k / \bar{\gamma}$. In Fig. 4 we compare it with the autocorrelation function obtained by stochastic simulations.

Here again, the autocorrelation function is monotonic which indicates the lack of oscillations. 
Fig. 4 Comparison of the autocorrelation function given in (25) (solid line) and the one obtained by stochastic simulations (dots). Inset shows graphs of the autocorrelation function given in (25) for $T \in[9,21]$. Parameters are fixed at $k=100, \gamma=4.1, \gamma_{d}=1$, $q_{1}=0.75, q_{2}=0.25, \tau_{1}=10$, $\tau_{2}=20$

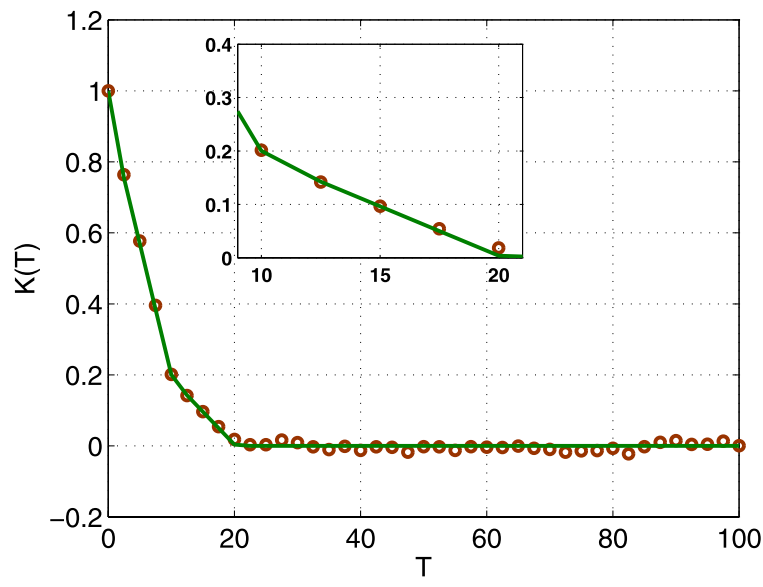

\subsection{Delayed Protein Degradation-the Full Model}

\subsubsection{Deterministic Description}

We now consider the classical model of gene expression presented in network (4) involving four biochemical processes: transcription, translation, mRNA, and protein degradation (Thattai and van Oudenaarden 2001). As in the previous section, we take into account that the process of protein degradation consists of several steps which take certain time and we model it by a single process with the time delay $\tau$ and the intensity $\gamma_{d}$ (we keep as before the possibility of an instantaneous degradation):

$$
\begin{aligned}
& \text { DNA } \stackrel{k_{r}}{\longrightarrow} \text { mRNA }, \quad \text { mRNA } \stackrel{\gamma_{r}}{\longrightarrow} \emptyset, \\
& \text { mRNA } \stackrel{k_{p}}{\longrightarrow} P, \quad P \stackrel{\gamma_{p}}{\longrightarrow} \emptyset, \quad P \stackrel{\gamma_{d}}{\longrightarrow} \emptyset .
\end{aligned}
$$

We extend (9a) and (9b) into the following system of differential equations:

$$
\begin{aligned}
& \frac{d x}{d t}=k_{r}-\gamma_{r} x(t), \\
& \frac{d z}{d t}=k_{p} x(t)-\left(\gamma_{p}+\gamma_{d}\right) z(t), \\
& \frac{d y}{d t}=k_{p} x(t)-\gamma_{p} z(t)-\gamma_{d} z(t-\tau),
\end{aligned}
$$

where $x, z$ and $y$ denote concentrations of mRNA, active proteins and all proteins, respectively. We take the initial condition of the type (8), that is $x_{0}=z_{0}(t)=y_{0}(t)=$ 0 for $t<0, x(0)=x^{0}$, and $z_{0}(0)=y_{0}(0)=z^{0}$. The system (27) can be easily solved. It turns out that solutions of system (27) are monotonic for $t$ large enough and tend to a steady state $\bar{x}=k_{r} / \gamma_{r}, \bar{z}=k_{p} k_{r} /\left(\left(\gamma_{p}+\gamma_{d}\right) \gamma_{r}\right), \bar{y}=\bar{z}\left(1+\gamma_{d} \tau\right)$. Moreover, $x$ is monotonic for all $t>0, z$ can exhibit at most one switch of monotonicity, and $y$ can exhibit at most three switches of monotonicity, see Fig. 5 and also the Supporting Information. Thus, also in this case no oscillations are possible. 

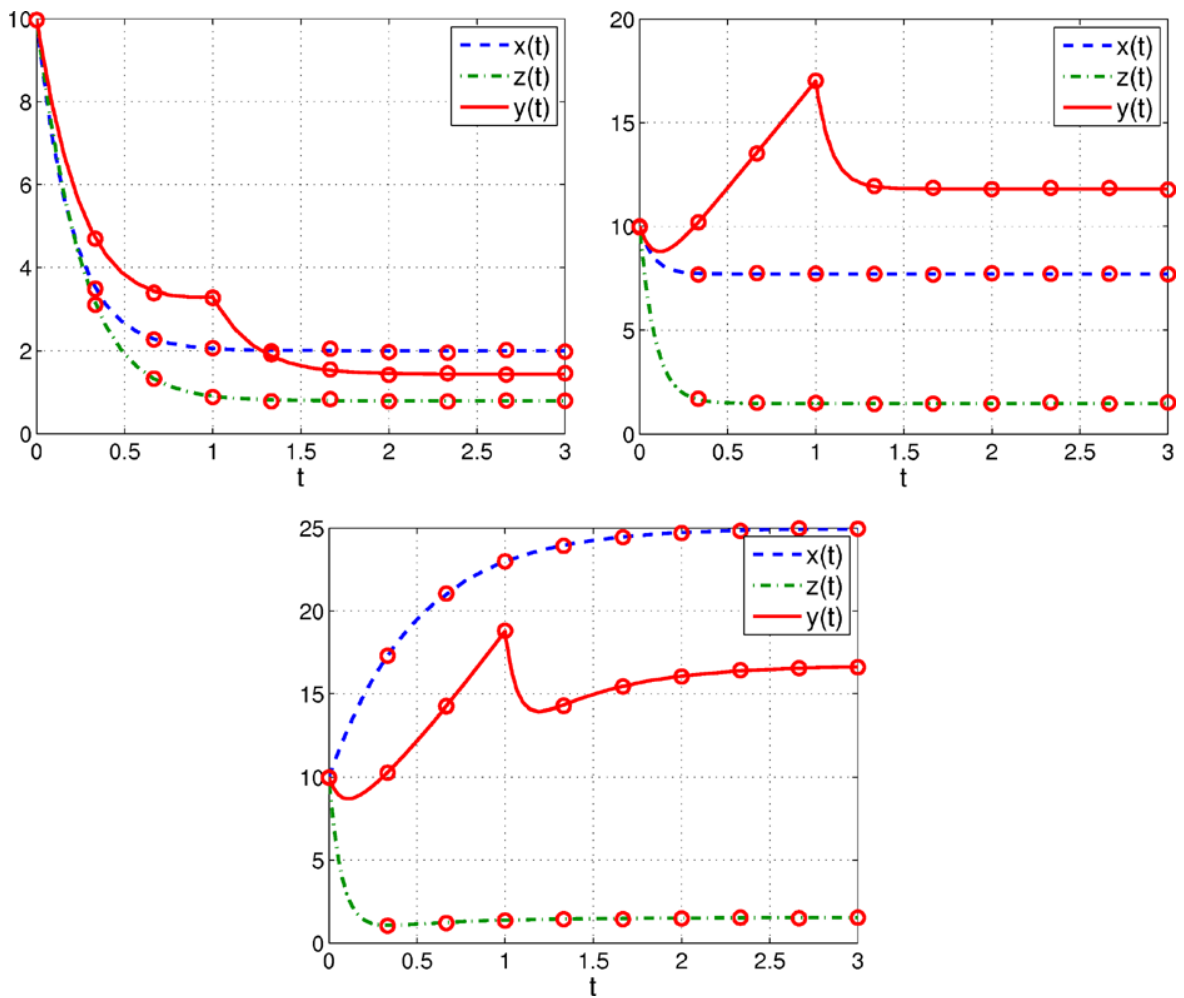

Fig. 5 Plots show different numbers of monotonicity switches in solutions of the system given in (27) for different values of parameters. At the upper left side, $x(t), y(t), z(t)$ do not change their monotonicity $\left(k_{r}=10, \gamma_{r}=5, k_{p}=2.3, \gamma_{p}=5, \gamma_{d}=0.8\right)$. At the upper right, $x(t), z(t)$ do not change their monotonicity and the function $y(t)$ changes its monotonicity twice $\left(k_{r}=100, \gamma_{r}=13, k_{p}=2.3, \gamma_{p}=5\right.$, $\left.\gamma_{d}=7\right)$. At the bottom, only $x(t)$ is monotonic, $z(t)$ changes its monotonicity once, and $y(t)$ changes its monotonicity three times $\left(k_{r}=50, \gamma_{r}=2, k_{p}=0.85, \gamma_{p}=4, \gamma_{d}=10\right)$. For all plots, the following parameters are fixed: $\tau=1, x^{0}=y^{0}=z^{0}=10$

\subsubsection{Stochastic Description}

In the stochastic approach we again pass from concentrations of substances to numbers of corresponding molecules. Let $r(t)$ be the number of mRNA molecules, $p(t)$ the number of active protein molecules at time $t, d(t)$ the number of delayed protein degradations initiated since the time $t=0$, and $y(t)$ the total number of protein molecules related to $p(t), d(t)$ and $d(t-\tau)$ as before in (14). The Master equation for the probability mass function $P(r, p, d, t)$ can be written it the following form:

$$
\begin{aligned}
\frac{d P(r, p, d, t)}{d t}= & k_{r}(P(r-1, p, d, t)-P(r, p, d, t)) \\
& +\gamma_{r}((r+1) P(r+1, p, d, t)-r P(r, p, d, t)) \\
& +k_{p}(r P(r, p-1, d, t)-r P(r, p, d, t)) \\
& +\gamma_{p}((p+1) P(r, p+1, d, t)-p P(r, p, d, t))
\end{aligned}
$$



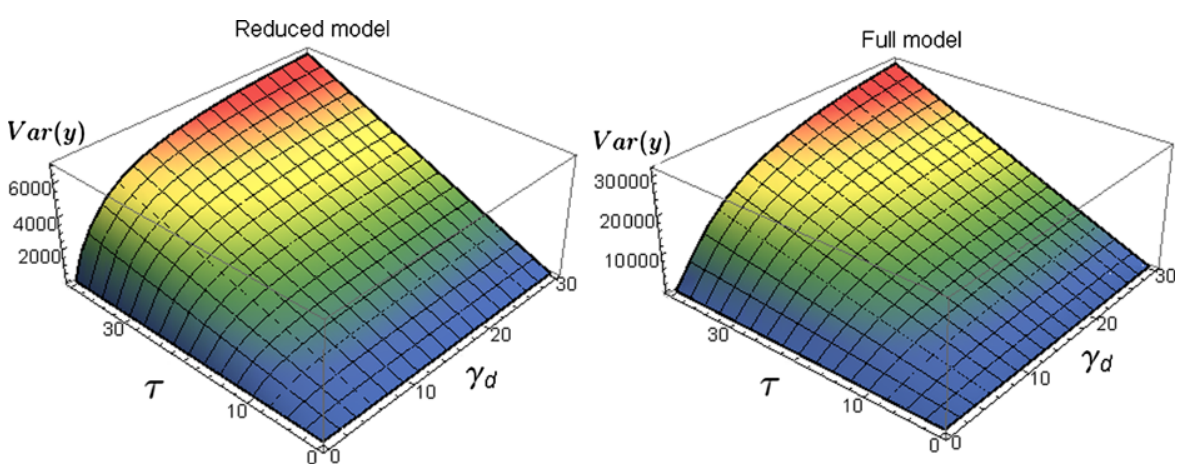

Fig. 6 The variance of the total number of molecules as a function of $\gamma_{d}$ and $\tau$ for the reduced model (6) (left) and full model (26) (right). For both models mean value of observed proteins is equal, that is we assume that $k=k_{r} k_{p} / \gamma_{r}$. Parameters are fixed at $k_{r}=100, \gamma_{r}=4, k_{p}=8, \gamma_{p}=4.1$

$$
+\gamma_{d}((p+1) P(n, p+1, d-1)-p P(r, p, d, t)) .
$$

Proceeding as before (see the Supporting Information) we get the expected value and the variance of $y$ in the stationary state. For the expected value we again get

$$
\langle y\rangle=\langle p\rangle\left(1+\gamma_{d} \tau\right) .
$$

The formula for the variance is rather complicated (see (2.26) in the Supporting Information). Here we present two asymptotic expressions:

$$
\operatorname{Var}(y)=\langle p\rangle\left(1+\gamma_{d} \tau+\frac{k_{p}\left(1+2 \gamma_{d} \tau\right)}{\gamma_{r}+\gamma_{p}+\gamma_{d}}\right)
$$

for small $\tau$ and

$$
\begin{aligned}
\operatorname{Var}(y)= & \operatorname{Var}(p)+2\langle p\rangle k_{p} \gamma_{d}\left(\frac{\gamma_{p}}{\gamma_{r}\left(\gamma_{p}+\gamma_{d}\right)^{2}}-\frac{\gamma_{d}}{\gamma_{r}^{2}\left(\gamma_{r}+\gamma_{p}+\gamma_{d}\right)}\right) \\
& +\langle p\rangle \gamma_{d}\left(1+2\langle p\rangle \frac{\gamma_{d}}{k_{r}} \tau\right)
\end{aligned}
$$

for large $\tau$; see also Fig. 6 for graphs of $\operatorname{Var}(y)$ as a function of $\gamma_{d}$ and $\tau$ for both reduced and full model.

The autocorrelation function for the full model was obtained numerically by stochastic simulations. Results are presented in Fig. 7. They indicate the lack of oscillations in the system.

\section{Discussion}

Cyclic behavior is ubiquitous in nature. It may have the form of circadian clocks or oscillations of smaller time periods. It is important to understand molecular causes of such behavior. It is known that negative feedbacks and time delays may cause 
Fig. 7 Autocorrelation functions for mRNA and protein $P$ levels obtained by stochastic simulations of reaction network (26). Inset shows graphs of those autocorrelation functions for $T \in[17,35]$. Parameters are fixed at $k_{r}=100, \gamma_{r}=13, k_{p}=2.3$, $\gamma_{p}=5, \gamma_{d}=7, \tau=20$

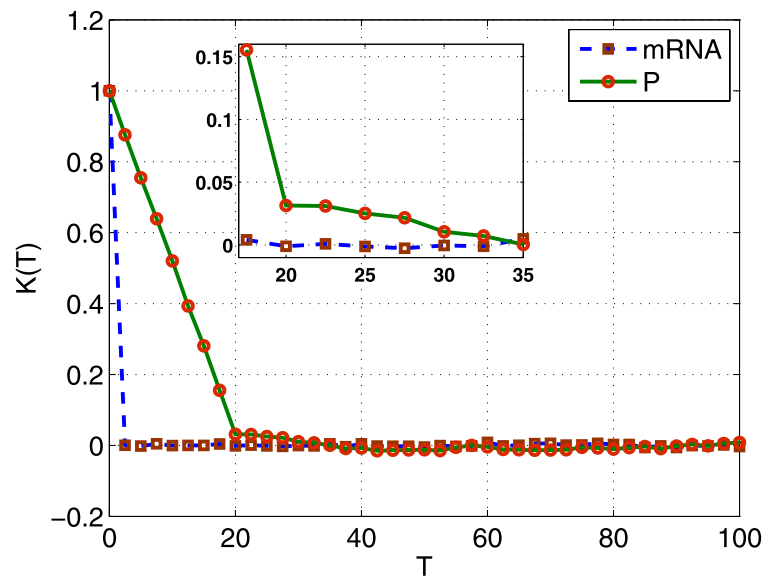

oscillations in time evolution of microscopic (genetic) and macroscopic (ecological) systems of living organisms. It was argued recently by Bratsun et al. (2005) that combined effects of time delay of protein degradation and stochasticity may cause an oscillatory behavior in simple models of gene expression. We show that this is not the case. We develop a general methodology to deal with time delays in biological systems and apply it to simple gene expression models. We prove that if we assume that a process of degradation is consuming that is molecules which started to degrade cannot take part in other processes, then oscillatory behavior is no longer present in such systems. It follows that the delayed protein degradation alone cannot cause oscillations. Therefore we should look for different mechanisms responsible for oscillations observed in biological experiments. To indicate the presence of oscillations in the reduced model of gene expression, the autocorrelation function and its power spectrum was computed in Bratsun et al. (2005) and Galla (2009). In both papers, the authors have not taken into account the consuming character of the degradation processes. We were able to compute analytically the autocorrelation function. Its monotonicity indicates the lack of oscillations in the system.

It would be very important to extend the methodology introduced in this paper to other systems with time delays such as delayed transcription and translation. In the case of translation, after protein molecules are produced, mRNA molecules are back into the system (as opposed to degradation processes). This makes such systems more complicated. Careful analytical analysis is needed here. It is especially important to study the effect of combined time delays and stochasticity on the behavior of such systems, and in particular on the possibility of stable oscillations.

Acknowledgements This work was supported by the Polish Ministry of Science and Higher Education under the grant N201 023 31/2069 (JM and JP) and N201 362536 (MB and UF).

Open Access This article is distributed under the terms of the Creative Commons Attribution Noncommercial License which permits any noncommercial use, distribution, and reproduction in any medium, provided the original author(s) and source are credited. 


\section{References}

Alberts, B., Johnson, A., Lewis, J., Raff, M., Roberts, K., \& Walter, P. (2002). Molecular biology of the cell, 4th ed. New York: Garland.

Alboszta, J., \& Miękisz, J. (2004). Stability of evolutionarily stable strategies in discrete replicator dynamics with time delay. J. Theor. Biol., 231, 175-179.

Barrio, M., Burrage, K., Leier, A., \& Tian, T. (2006). Oscillatory regulation of Hes1: discrete stochastic delay modelling and simulation. PLoS Comput. Biol., 2, 1017-1030.

Berg, O. (1978). A model for the statistical fluctuations of protein numbers in a microbial population. J. Theor. Biol., 71, 587-603.

Bernard, S., Čajavec, B., Pujo-Menjouet, L., Mackey, M., \& Herzel, H. (2006). Modelling transcriptional feedback loops: the role of Gro/TLE1 in Hes1 oscillations. Philos. Trans. R. Soc. Lond. A, 364, 1155-1170.

Bodnar, M. (2000). The nonnegativity of solutions of delay differential equations. Appl. Math. Lett., 13, 91-96.

Bratsun, D., Volfson, D., Tsimring, L., \& Hasty, J. (2005). Delay-induced stochastic oscillations in gene regulation. Proc. Natl. Acad. Sci. USA, 102, 14593-14598.

Cai, X. (2007). Exact stochastic simulation of coupled chemical reactions with delays. J. Chem. Phys., $126,124108$.

Clayton, C., \& Shapira, M. (2007). Post-transcriptional regulation of gene expression in trypanosomes and leishmanias. Mol. Biochem. Parasitol., 156, 93-101.

Erneux, T. (2009). Applied delay differential equations. New York: Springer.

Foryś, U. (2004). Biological delay systems and the Mikhailov criterion of stability. J. Biol. Syst., 12, 1-16.

Galla, T. (2009). Intrinsic fluctuations in stochastic delay systems: Theoretical description and application to a simple model of gene regulation. Phys. Rev. E, 80, 21909-21918.

Gillespie, D. (1977). Exact stochastic simulation of coupled chemical reactions. J. Phys. Chem., 81, 23402361.

Gopalsamy, K. (1992). Stability and oscillations in delay differential equations of population dynamics. Dordrecht: Kluwer Academic.

Guillouzic, S., L'Heureux, I., \& Longtin, A. (1999). Small delay approximation of stochastic delay differential equations. Phys. Rev. E, 59, 3970-3982.

Györi, I., \& Ladas, G. (1991). Oscillation theory of delay differential equations with applications. Oxford: Clarendon.

Hale, J. (1997). Theory of functional differential equations. New York: Springer.

Hornos, J., Schultz, D., Innocentini, G., Wang, J., Walczak, A., Onuchic, J., \& Wolynes, P. (2005). Selfregulating gene: an exact solution. Phys. Rev. E, 72, 51907-51912.

Jensen, M., Sneppen, K., \& Tiana, G. (2003). Sustained oscillations and time delays in gene expression of protein Hes1. FEBS Lett., 541, 176-177.

Kepler, T., \& Elston, T. (2001). Stochasticity in transcriptional regulation: origins, consequences, and mathematical representations. Biophys. J., 81, 3116-3136.

Komorowski, M., Miękisz, J., \& Kierzek, A. (2009). Translational repression contributes greater noise to gene expression than transcriptional repression. Biophys. J., 96, 372-384.

Krishna, S., Jensen, M., \& Sneppen, K. (2006). Minimal model of spiky oscillations in NF- $\kappa$ B signaling. Proc. Natl. Acad. Sci. USA, 103, 10840-10845.

Kuang, Y. (1993). Delay. differential equations: with applications in population dynamics. New York: Academic Press.

Küchler, U., \& Mensch, B. (1992). Langevins stochastic differential equation extended by a time-delayed term. Stoch. Stoch. Rep., 40, 23-42.

Lei, J., \& Mackey, M. (2007). Stochastic differential delay equation, moment stability, and application to hematopoietic stem cell regulation system. SIAM J. Appl. Math., 67, 387-407.

Lewis, J. (2003). Autoinhibition with transcriptional delay: A simple mechanism for the zebrafish somitogenesis oscillator. Curr. Biol., 13, 1398-1408.

Liu, Y., Loros, J., \& Dunlap, J. C. (2000). Phosphorylation of the neurospora clock protein frequency determines its degradation rate and strongly influences the period length of the circadian clock. Proc. Natl. Acad. Sci. USA, 97, 234-239.

Mao, X., Yuan, C., \& Zou, J. (2005). Stochastic differential delay equations of population dynamics. J. Math. Anal. Appl., 304, 296-320.

Marquez-Lago, T., Leier, A., \& Burrage, K. (2010). Probability distributed time delays: integrating spatial effects into temporal models. BMC Syst. Biol., 4, 19-35. 
Mather, W., Bennett, M., Hasty, J., \& Tsimring, L. (2009). Delay-induced degrade-and-fire oscillations in small genetic circuits. Phys. Rev. Lett., 102, 068105.

McAdams, H., \& Arkin, A. (1997). Stochastic mechanisms in gene expression. Proc. Natl. Acad. Sci. USA, 94, 814-819.

Melzner, I. et al. (2006). Biallelic deletion within 16p13. 13 including SOCS-1 in Karpas1106P mediastinal B-cell lymphoma line is associated with delayed degradation of JAK2 protein. Int. J. Cancer, 118, 1941-1944.

Miękisz, J. (2008). Evolutionary game theory and population dynamics. In Capasso, V. \& Lachowicz, M. (Eds.), Lecture notes in mathematics: Vol. 1940. Multiscale problems in the life sciences, from microscopic to macroscopic (pp. 269-316).

Monk, N. (2003). Oscillatory expression of Hes1, p53, and NF- $\kappa$ B driven by transcriptional time delays. Curr. Biol., 13, 1409-1413.

Ohira, T., \& Milton, J. (1995). Delayed random walks. Phys. Rev. E, 52, 3277-3280.

Ohira, T., \& Yamane, T. (2000). Delayed stochastic systems. Phys. Rev. E, 61, 1247-1257.

Paulsson, J. (2004). Summing up the noise in gene networks. Nature, 427, 415-418.

Paulsson, J. (2005). Models of stochastic gene expression. Phys. Life Rev., 2, 157-175.

Ribeiro, A. (2010). Stochastic and delayed stochastic models of gene expression and regulation. Math. Biosci., 223, 1-11.

Roussel, M. R., \& Zhu, R. (2006). Validation of an algorithm for delay stochastic simulation of transcription and translation in prokaryotic gene expression. Phys. Biol., 3, 274-284.

Schlicht, R., \& Winkler, G. (2008). A delay stochastic process with applications in molecular biology. J. Math. Biol., 57, 613-648.

Smolen, P., Baxter, D. A., \& Byrne, J. H. (2001). Modeling circadian oscillations with interlocking positive and negative feedback loops. J. Neurosci., 21, 6644-6656.

Sriram, K., \& Gopinathan, M. S. (2004). A two variable delay model for the circadian rhythm of neurospora crassa. J. Theor. Biol., 231, 23-38.

Swain, P., Elowitz, M., \& Siggia, E. (2002). Intrinsic and extrinsic contributions to stochasticity in gene expression. Proc. Natl. Acad. Sci. USA, 99, 12795-12800.

Thattai, M., \& van Oudenaarden, A. (2001). Intrinsic noise in gene regulatory networks. Proc. Natl. Acad. Sci. USA, 98, 8614-8619.

Tian, T., Burrage, K., Burrage, P., \& Carletti, M. (2007). Stochastic delay differential equations for genetic regulatory networks. J. Comput. Appl. Math., 205, 696-707.

Tiana, G., Krishna, S., Pigolotti, S., Jensen, M., \& Sneppen, K. (2007). Oscillations and temporal signalling in cells. Phys. Biol., 4, R1-R17.

Timmer, J., Müller, T., Swameye, I., Sandra, O., \& Klingmüller, U. (2004). Modeling the nonlinear dynamics of cellular signal transduction. Int. J. Bifurc. Chaos Appl. Sci. Eng., 14, 2069-2079.

Van Kampen, N. (1997). Stochastic processes in physics and chemistry, 2nd ed. Amsterdam: Elsevier. 